\title{
Critical exponents and universality for the isotropic-nematic phase transition in a system of self-assembled rigid rods on a lattice
}

\author{
L. G. López, D. H. Linares, and A. J. Ramirez-Pastor* \\ Departamento de Física, Instituto de Física Aplicada, CONICET, Universidad Nacional de San Luis, 5700 San Luis, Argentina
}

(Received 10 March 2009; revised manuscript received 1 October 2009; published 22 October 2009)

\begin{abstract}
Monte Carlo simulations have been carried out for a system of monomers on square lattices that, by decreasing temperature or increasing density, polymerize reversibly into chains with two allowed directions and, at the same time, undergo a continuous isotropic-nematic (IN) transition. The results show that the self-assembly process affects the nature of the transition. Thus, the calculation of the critical exponents and the behavior of Binder cumulants indicate that the universality class of the IN transition changes from twodimensional Ising-type for monodisperse rods without self-assembly to $q=1$ Potts-type for self-assembled rods.

DOI: 10.1103/PhysRevE.80.040105

PACS number(s): 05.50.+q, 64.75.Yz, 61.20.Ja, 64.70.mf
\end{abstract}

Self-assembly is a challenging field of research driven principally by the desire to design new materials. Moreover, self-assembly is used permanently in biological systems to construct supramolecular structures such as virus capsids, filaments, and many others large molecular complexes. So, understanding the rules of self-assembly has important applications to both materials science and biology [1].

On the other hand, the isotropic-nematic (IN) transition in solutions of rodlike particles has been attracting a great deal of interest since long ago. A seminal contribution to this subject was made by Onsager [2] with his paper on the IN transitions of infinitely thin rods. This theory shows that particles interacting with only excluded volume interaction may exhibit a rich phase diagram despite the absence of any attraction. Later, computer simulations of hard ellipses of finite length [3] confirmed the Onsager prediction that particle shape anisotropy can be a sufficient condition to induce the long-range orientational order found in nematic liquid crystals.

In contrast to ordinary liquid crystal, many rodlike biological polymers are formed by monomers reversibly selfassembling into chains of arbitrary length so that these systems exhibit a broad equilibrium distribution of filament lengths. An experimental contribution to the study of these systems has been presented by Viamontes et al. [4]. The authors reported a continuous IN transition for solutions of long F-actin (average filament length longer than $2 \mu \mathrm{m}$ ) and showed the existence of a first-order phase separation for solutions of F-actin with average filament length shorter than $2 \mu \mathrm{m}$. These findings contradict what is generally accepted in the literature: in three dimensions, the IN transition is typically first order. On the other hand, in two dimensions both continuous [5] and first-order [6] IN transitions can occur. Here, we consider a self-assembled two-dimensional (2D) system that undergo a IN transition, which is expected to be a continuous phase transition [7].

As mentioned above, the self-assembled system is intrinsically polydisperse. While being able to solve explicitly only the monodisperse case, Onsager [2] already outlined the possible extension of the theory to polydisperse systems. In

\footnotetext{
*antorami@unsl.edu.ar
}

this line of work, a detailed investigation of the effects of full length polydispersity, i.e., of a continuous distribution of rod lengths, on the Onsager theory has been recently developed by Speranza and Sollich [8]. Another approach to the problem of monodisperse rodlike mixtures has been proposed by Zwanzig [9]. The Zwanzig model has been also extended to polydisperse systems [10], providing thus a useful starting point for understanding the effects of polydispersity on the phase behavior of hard rod systems. However, a complete description of a system of self-assembled rods should consider not only the effects of polydispersity but also the influence of the polymerization process.

In this context, we focus on a system composed of monomers with two attractive (sticky) poles that polymerize reversibly into polydisperse chains and, at the same time, undergo a continuous phase transition. So, the interplay between the self-assembly process and the nematic ordering is a distinctive characteristic of these systems.

The same system has been recently considered by Tavares et al. [7]. Using an approach in the spirit of the Zwanzig model, the authors studied the IN transition occurring in a two-dimensional system of self-assembled rigid rods. The obtained results revealed that nematic ordering enhances bonding. In addition, the average rod length was described quantitatively in both phases, while the location of the ordering transition, which was found to be continuous, was predicted semiquantitatively by the theory.

Despite these interesting results there is an open question to be answered: "what type of phase transition is it?" Tavares et al. [7] assumed as working hypothesis that the nature of the IN transition remains unchanged with respect to the case of monodisperse rigid rods on square lattices, where the transition is in the 2D Ising universality class [11,12]. In this context, the confirmation (or not) of this hypothesis is not only important to resolve the universality class of the IN transition occurring in a system of self-assembled rods but also to shed light on our understanding of the effect of the self-assembly process on the nature of the transition. The objective of this Rapid Communication is to provide a thorough study in this direction. For this purpose, extensive Monte Carlo (MC) simulations supplemented by analysis using finite-size scaling (FSS) theory [13] have been carried out to study the critical behavior in a system of self- 
assembled rigid rods deposited on square lattices with two allowed directions. The calculations were developed at constant temperature and different densities, thus allowing a direct comparison with previous results for long monodisperse rigid rods on two-dimensional lattices $[11,12]$. Then, the conventional normalized scaling variable $\varepsilon \equiv T / T_{c}-1$ was replaced by $\varepsilon \equiv \theta / \theta_{c}-1$, where $T, T_{c}, \theta$, and $\theta_{c}$ represent temperature, critical temperature, density, and critical density, respectively. A nematic phase, characterized by a big domain of parallel self-assembled rigid rods, is separated from the disordered state by a continuous IN transition occurring at a finite critical density. The results show that the self-assembly process affects the nature of the transition. Thus, the determination of the critical exponents indicates that the universality class of the IN transition changes from 2D Ising-type for monodisperse rods without self-assembly [12] to $q=1$ Potts-type for self-assembled rods.

In a recent paper, Fischer and Vink [14] indicated that the transition studied in Refs. [11,12] corresponds to a liquid-gas transition rather than IN. This interpretation is consistent with the 2D-Ising critical behavior observed for monodisperse rigid rods on square lattices. However, as mentioned in the previous paragraph, the universality class of selfassembled rods changes from that of the 2D Ising model and the result in Ref. [14] is not generalizable to the system studied here. Accordingly, we will continue using the term "IN phase transition" as in the previous work of Tavares et al. [7].

As in Ref. [7], we consider a system of self-assembled rods with a discrete number of orientations in $2 \mathrm{D}$. We assume that the substrate is represented by a square lattice of $M=L \times L$ sites with periodic boundary conditions. $N$ particles are adsorbed on the substrate with two possible orientations along the principal axis of the lattice. These particles interact with nearest neighbors (NN) through anisotropic attractive interactions. Then the adsorbed phase is characterized by the Hamiltonian $H=\sum_{\langle i, j\rangle} w_{i j} c_{i} c_{j}$, where $\langle i, j\rangle$ indicates a sum over $\mathrm{NN}$ sites; $w_{i j}$ represents the NN lateral interaction, which is $w_{i j}=-w$ if two neighboring particles $i$ and $j$ are aligned with each other and with the intermolecular vector and is $w_{i j}=0$ otherwise; and $c_{i}$ is the occupation variable with $c_{i}=0$ if the site $i$ is empty, and $c_{i}=1$ if the site $i$ is occupied.

A cluster or uninterrupted sequence of bonded particles is a self-assembled rod. At fixed temperature, the average rod length increases as the density increases and the polydisperse rods will undergo a nematic ordering transition [7]. In order to follow the formation of the nematic phase from the isotropic phase, we use the order parameter defined in Ref. [7], which can be written as $\delta=\left|N_{h}-N_{v}\right| /\left(N_{h}+N_{v}\right)$, where $N_{h}\left(N_{v}\right)$ is the number of particles in clusters aligned along the horizontal (vertical) direction. When the system is disordered, all orientations are equivalents and $\delta$ is zero. In the critical regime, the particles align along one direction and $\delta$ is different from zero. In other words, the IN phase transition is accomplished by a breaking of the orientational symmetry and $\delta$ appears as a proper order parameter to elucidate this phenomenon.

The problem has been studied by canonical Monte Carlo simulations using an vacancy-particle-exchange Kawasaki dynamics [15] and Metropolis acceptance probability [16]. Typically, the equilibrium state can be well reproduced after discarding the first $5 \times 10^{6}$ Monte Carlo steps (MCS). Then, the next $6 \times 10^{8} \mathrm{MCS}$ are used to compute averages.

In our Monte Carlo simulations, we set the temperature $T$, varied the density $\theta=N / M$, and monitored the order parameter $\delta$, which can be calculated as simple average. The quantities related with the order parameter, such as the susceptibility $\chi$, and the reduced fourth-order cumulant $U_{L}$ introduced by Binder [13] were calculated as $\chi=L^{2} / k_{B} T\left[\left\langle\delta^{2}\right\rangle-\langle\delta\rangle^{2}\right]$ and $U_{L}=1-\left\langle\delta^{4}\right\rangle /\left[3\left\langle\delta^{2}\right\rangle^{2}\right]$, where $\langle\cdots\rangle$ means the average over the MC simulation runs. In addition, in order to discuss the nature of the phase transition, the fourth-order energy cumulant $U_{E}$ was obtained as $U_{E}=1-\left\langle H^{4}\right\rangle /\left[3\left\langle H^{2}\right\rangle^{2}\right]$.

The critical behavior of the present model has been investigated by means of the computational scheme described in the previous paragraphs and finite-size scaling analysis. The FSS theory implies the following behavior of $\delta, \chi$, and $U_{L}$ at criticality: $\delta=L^{-\beta / \nu} \widetilde{\delta}\left(L^{1 / \nu} \varepsilon\right), \quad \chi=L^{\gamma / \nu} \widetilde{\chi}\left(L^{1 / \nu} \varepsilon\right)$, and $U_{L}=\widetilde{U}_{L}\left(L^{1 / \nu} \varepsilon\right)$ for $L \rightarrow \infty, \varepsilon \rightarrow 0$ such that $L^{1 / \nu} \varepsilon=$ finite, where $\varepsilon \equiv \theta / \theta_{c}-1$. Here $\beta, \gamma$, and $\nu$ are the standard critical exponents of the order parameter, susceptibility, and correlation length, respectively. $\tilde{\delta}, \tilde{\chi}$ and $\tilde{U}_{L}$ are scaling functions for the respective quantities.

The phase diagram of the system under study has been recently reported by Tavares et al. [7]. The authors showed that the critical density, at which the IN transition occurs, increases monotonically as $k_{B} T / w$ is increased. Thus, the nematic phase is stable at low temperatures and high densities [see Fig. 1(a) in Ref. [7]]. In addition, Tavares et al. found strong numerical evidence that the IN transition is a continuous phase transition. However, the authors were not able to determine the critical quantities characterizing the universality class of the mentioned transition. In the following, we try to resolve this problem.

In our study and based on the phase diagram given in Ref. [7], we set the lateral interaction to $w=4 k_{B} T$. With this value of $w$, it is expected the appearance of a nematic phase at intermediate densities. Accordingly, the density was varied between 0.4 and 0.6. For each value of $\theta$, the effect of finite size was investigated by examining square lattices with $L=60,80,100$, and 120 .

We start with the calculation of the order parameter (Fig. 1), susceptibility (inset in Fig. 1), and cumulant (Fig. 2) plotted versus $\theta$ for several lattice sizes. In the vicinity of the critical point, cumulants show a strong dependence on the system size. However, at the critical point the cumulants adopt a nontrivial value $U^{*}$; irrespective of system sizes in the scaling limit. Thus, plotting $U_{L}(\theta)$ for different linear dimensions yields an intersection point $U^{*}$, which gives an accurate estimation of the critical density in the infinite system and allows us to make a preliminary identification of the universality class of the transition [13]. In this case, the values obtained for the critical density and the intersection point of the cumulants were $\theta_{c}=0.524(4)$ and $U^{*}=0.639(3)$, respectively. This fixed value of the cumulants has changed from that obtained for monodisperse rigid rods on square lattices $\left[U^{*}=0.615(5)\right]$, which may be taken as a first indica- 


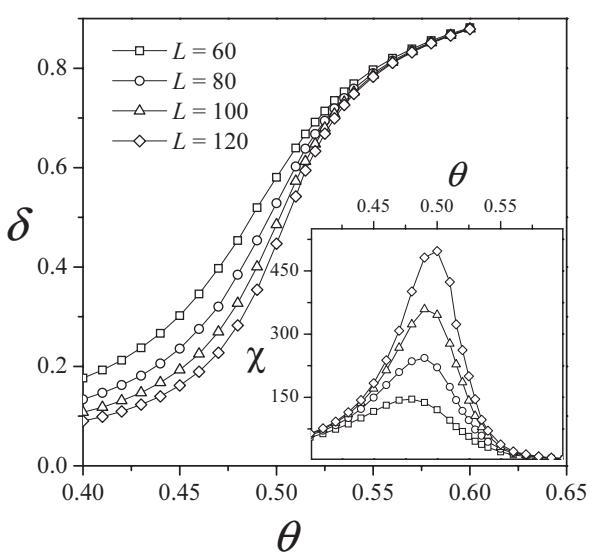

FIG. 1. Size dependence of the order parameter $(\delta)$ as a function of density $(\theta)$. Inset: size dependence of the susceptibility $(\chi)$ as a function of density $(\theta)$.

tion that the universality class of the present model is different from the well-known 2D Ising-type for monodisperse rods [12]. In the lower-right inset, the data are plotted over a wider range of densities. As can be seen, the curves exhibit the typical behavior of $U_{L}$ in the presence of a continuous phase transition. Namely, the order-parameter cumulant shows a smooth increase from 0 to $2 / 3$ instead of the characteristic deep (negative) minimum, as in a first-order phase transition [13].

In order to discard the possibility that the phase transition is a first-order one, the energy cumulants have been measured for different lattice sizes ranging between $L=20$ and $L=80$. As is well known, the finite-size analysis of $U_{E}$ is a simple and direct way to determine the order of a phase transition [13]. Our results for $U_{E}$ show a dip close to the critical density $\theta_{c}=0.524$ for all system sizes, but this minimum scales to $2 / 3$ in the thermodynamic limit as can be seen in the upper-left inset of Fig. 2. These results exclude a firstorder transition, confirming the predictions by Tavares et al. [7].

Next, the critical exponents will be calculated. As stated in Ref. [17], the critical exponent $\nu$ can be obtained by con-

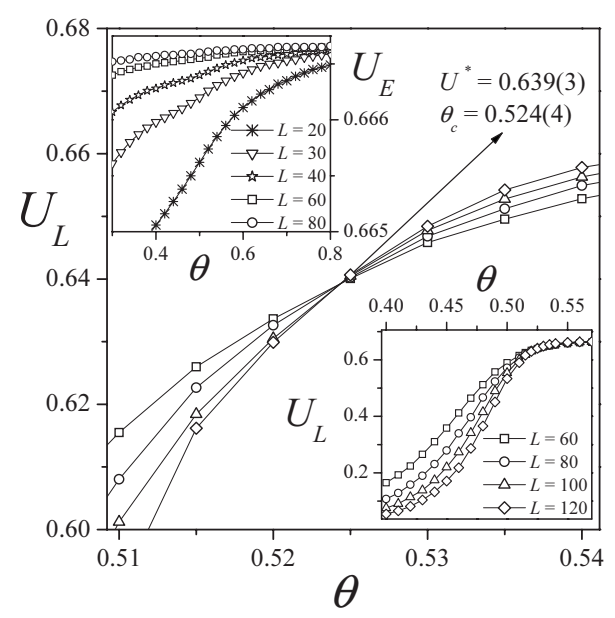

FIG. 2. Curves of $U_{L}(\theta)$ vs $\theta$ for square lattices of different sizes. From their intersections one obtained $\theta_{c}$. In the inset, the data are plotted over a wider range of densities.



FIG. 3. (a) Log-log plot of the size dependence of the maximum values of derivatives of various thermodynamic quantities used to determine $\nu$. (b) Log-log plot of the size dependence of the maximum value of the susceptibility, the point of inflection of the order parameter and the maximum value of the derivative of the order parameter used to determine $\gamma$ and $\beta$, respectively.

sidering the scaling behavior of certain thermodynamic derivatives with respect to the density $\theta$, for example, the derivative of the cumulant and the logarithmic derivatives of $\langle\delta\rangle$ and $\left\langle\delta^{2}\right\rangle$. In Fig. 3(a), we plot the maximum value of these derivatives as a function of system size on a log-log scale. The results for $1 / \nu$ from these fits are given in Fig. 3(a). Combining these three estimates, we obtain $\nu=1.33(1)$. Once we know $\nu$, the exponent $\gamma$ can be determined by scaling the maximum value of the susceptibility [17]. Our data for $\left.\chi\right|_{\max }$ are shown in Fig. 3(b). The value obtained for $\gamma$ is $\gamma=2.36(4)$.

On the other hand, the standard way to extract the exponent ratio $\beta / \nu$ is to study the scaling behavior of $\langle\delta\rangle$ at the point of inflection $\left(\left.\langle\delta\rangle\right|_{\text {inf }}\right)$, i.e., at the point where $d\langle\delta\rangle / d \theta$ is maximal. The scaling of $\left.\langle\delta\rangle\right|_{\text {inf }}$ is shown in Fig. 3(b). The linear fit through all data points gives $\beta^{\left(\left.\langle\delta\rangle\right|_{\text {inf }}\right)}=0.139(12)$. In the case of $d\langle\delta\rangle /\left.d \theta\right|_{\max }$ [see Fig. 3(b)], the value obtained from the fit is $\beta^{\left(d\langle\delta\rangle /\left.d \theta\right|_{\max }\right)}=0.138$ (3). Combining the two estimates, we obtain the final value $\beta=0.139(6)$.

The values calculated for $\nu, \beta$, and $\gamma$ clearly indicate that the IN phase transition belongs to the $q=1$ Potts universality class (ordinary percolation) [18]. This finding is also consistent with the crossing point of the cumulants shown in Fig. 2, which is in agreement with recent simulations on $2 \mathrm{D}$ site percolation by Vink [19], where a value of $U^{*} \approx 0.638$ has been obtained.

The universality observed in this study can be interpreted by analyzing the connection between the thermal phase transition (IN phase transition) occurring in the system and the behavior of the clusters of aligned monomers. In fact, preliminary results (based on the observation of the adsorbed state at critical regime) suggest that, at intermediate concentrations, the appearance of nematic order at critical density is 




FIG. 4. Data collapsing of the order parameter, $\delta L^{\beta / \nu}$ vs $|\varepsilon| L^{1 / \nu}$. The plot was made using $\theta_{c}=0.524$ and the exact percolation exponents $\nu=4 / 3$ and $\beta=5 / 36$.

accompanied by the simultaneous presence of a cluster of aligned monomers connecting the extremes of the lattice. In these conditions, an exact correspondence is expected between (1) the nematic order parameter and the infinite cluster; (2) the susceptibility and the mean cluster size; and (3) the IN phase transition and the percolation phase transition. A similar behavior has been observed for the site-diluted quenched Ising model at the percolation threshold [20].

The results are also consistent with previous research on polymer systems in 2D such as polymer networks [21] and branched polymers [22], which were shown to be in the universality class of ordinary percolation. Furthermore, the study of linear segments of size $k$ and $k$-mers of different structures and forms deposited on 2D regular lattices has demonstrated that the system, in all cases, belongs to the random percolation universality class [23].

The scaling behavior can be further tested by plotting $\langle\delta\rangle L^{\beta / \nu}$ vs $|\varepsilon| L^{1 / \nu}, \chi L^{-\gamma / \nu}$ vs $\varepsilon L^{1 / \nu}$, and $U$ vs $|\varepsilon| L^{1 / \nu}$ and looking for data collapsing. Using $\theta_{c}=0.524$ and the exact values

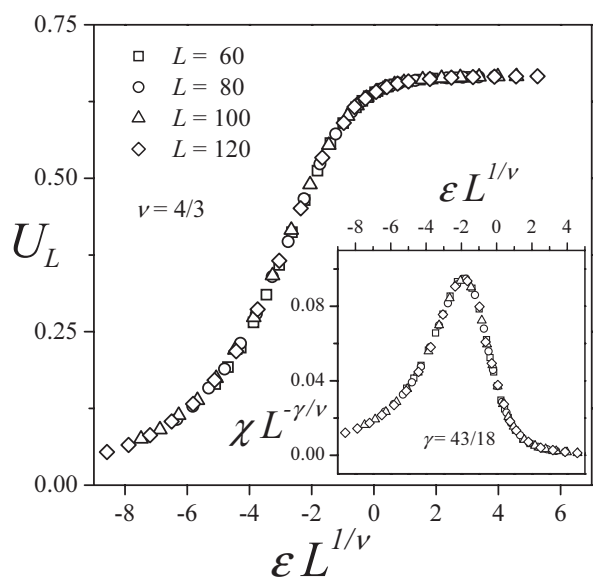

FIG. 5. Data collapsing of the cumulant, $U_{L}$ vs $\varepsilon L^{1 / \nu}$, and of the susceptibility, $\chi L^{-\gamma / \nu}$ vs $\varepsilon L^{1 / \nu}$ (inset). The plots were made using $\theta_{c}=0.524$ and exact percolation exponents $\nu=4 / 3$ and $\beta=43 / 18$.

of the critical exponents of the ordinary percolation $\nu=4 / 3$, $\beta=5 / 36$, and $\gamma=43 / 18$, we obtain an excellent scaling collapse as it is shown in Figs. 4 and 5. This Rapid Communication leads to independent controls and consistency checks of the values of all the critical exponents.

In summary, we have used Monte Carlo simulations and finite-size scaling theory to resolve the nature and universality class of the IN phase transition occurring in a model of self-assembled rigid rods. The existence of a continuous phase transition was confirmed. In addition, as was evident from our results, the self-assembly process affects the universality of the IN transition. Thus, the accurate determination of the critical exponents along with the behavior of Binder cumulants revealed that the universality class of the IN transition changes from 2D Ising-type for monodisperse rods without self-assembly to $q=1$ Potts-type for selfassembled rods.
[1] R. F. Service, Science 309, 95 (2005).

[2] L. Onsager, Ann. N.Y. Acad. Sci. 51, 627 (1949).

[3] J. Vieillard-Baron, J. Chem. Phys. 56, 4729 (1972).

[4] J. Viamontes, P. W. Oakes, and J. X. Tang, Phys. Rev. Lett. 97, 118103 (2006).

[5] D. Frenkel and R. Eppenga, Phys. Rev. A 31, 1776 (1985).

[6] R. L. C. Vink, Phys. Rev. Lett. 98, 217801 (2007).

[7] J. M. Tavares, B. Holder, and M. M. Telo da Gama, Phys. Rev. E 79, 021505 (2009).

[8] A. Speranza and P. Sollich, Phys. Rev. E 67, 061702 (2003).

[9] R. Zwanzig, J. Chem. Phys. 39, 1714 (1963).

[10] N. Clarke et al., J. Chem. Phys. 113, 5817 (2000).

[11] A. Ghosh and D. Dhar, EPL 78, 20003 (2007).

[12] D. A. Matoz-Fernandez, D. H. Linares, and A. J. RamirezPastor, EPL 82, 50007 (2008); J. Chem. Phys. 128, 214902 (2008).

[13] K. Binder, Applications of the Monte Carlo Method in Statistical Physics: Topics in Current Physics (Springer, Berlin, 1984).
[14] T. Fischer and R. L. C. Vink, EPL 85, 56003 (2009).

[15] K. Kawasaki, in Phase Transitions and Critical Phenomena, edited by C. Domb and M. S. Green (Academic, London, 1972).

[16] N. Metropolis, A. W. Rosenbluth, M. N. Rosenbluth, A. H. Teller, and E. Teller, J. Chem. Phys. 21, 1087 (1953).

[17] A. M. Ferrenberg and D. P. Landau, Phys. Rev. B 44, 5081 (1991); W. Janke, M. Katoot, and R. Villanova, ibid. 49, 9644 (1994); K. Binder and E. Luijten, Phys. Rep. 344, 179 (2001).

[18] F. Y. Wu, Rev. Mod. Phys. 54, 235 (1982).

[19] R. L. C. Vink (private communication).

[20] D. Stauffer and A. Aharony, Introduction to Percolation Theory (Taylor \& Francis, London, 1994).

[21] Y. Wu, B. Schmittmann, and R. K. P. Zia, J. Phys. A 41, 025004 (2008)

[22] A. Bunde, S. Havlin, and M. Porto, Phys. Rev. Lett. 74, 2714 (1995).

[23] V. Cornette, A. J. Ramirez-Pastor, and F. Nieto, Eur. Phys. J. B 36, 391 (2003); Physica A 327, 71 (2003). 